\title{
Diagnostic rigid and flexible oesophagoscopy in carcinoma of the oesophagus: a comparison
}

\author{
A J Ritchie, J McGuigan, K McManus, H M Stevenson, J R P Gibbons
}

\begin{abstract}
Background Flexible oesophagoscopy is regarded as superior to rigid oesophagoscopy on the basis of perforation rates as an end point. This advantage may be more apparent than real because no comparison has been made in a diagnostic setting in patients with carcinoma of the oesophagus with both perforation rate and diagnostic efficacy as indices.

Methods A retrospective analysis was carried out on data on 336 diagnostic oesophagoscopies in patients with carcinoma of the oesophagus, comparing rigid with flexible oesophagoscopy.

Results Both rigid and flexible oesophagoscopies were performed without perforation when they were used for diagnosis only. Rigid biopsy achieved a diagnostic success rate of $99.3 \%$, compared with $80.5 \%$ for flexible oesophagoscopy.

Conclusions Diagnostic oesophagoscopy can be achieved without perforation with either instrument, but the chance of diagnosing carcinoma was significantly greater with the rigid instrument.
\end{abstract}

(Thorax 1993;48:115-118)

Oesophagoscopy remains the most reliable investigative procedure in the diagnosis of cancer of the oesophagus. ${ }^{1}$ Dilatation, insertion of an oesophageal tube, and removal of foreign bodies have expanded the role of therapeutic procedures. Increasingly, fibreoptic instruments are regarded as superior to the rigid oesophagoscope in both diagnostic and therapeutic roles. ${ }^{2-5}$ Perforation is a hazard of oesophagoscopy irrespective of the type of instrument used ${ }^{6}$ and is a serious event that is frequently fatal. ${ }^{7}$ As an objective measure or end point, perforation is used in claiming an important advantage for the fibreoptic oesophagoscope over the rigid instrument, perforation rates ranging from $0.018 \%$ to $0.03 \%^{7}$ compared with $0.2 \%$ to $13 \%$ for the rigid instrument. ${ }^{9-11}$ Successful biopsy and diagnosis is another objective measure by which the two instruments might be compared, but there is little published material that directly compares the two in a similar setting or group of patients. ${ }^{2}$ The advantage of the flexible over the rigid instrument may be more apparent than real as there has been no prospective randomised clinical trial comparing the instruments in a diagnostic as opposed to therapeutic setting. Reports that claim an advantage for flexible oesophagoscopy have included many low risk patients, ${ }^{2}$ some without malignant disease, ${ }^{12}$ or in whom a normal oesophagus has been studied, ${ }^{8911}$ and have failed to appreciate the increased risk associated with therapeutic use of the rigid instrument. Endoscopic perforation of malignant strictures of the oesophagus is a recognised hazard and is not comparable to intubation in the normal oesophagus, a hazard increased by attempts at dilatation in elderly high risk patients, many of whom have inoperable disease and are debilitated and undergoing palliative procedures.

In a retrospective analysis we have compared the flexible and rigid oesophagoscopes in patients with carcinoma of the oesophagus, using the objective measures of rate of perforation and successful diagnosis in a series of patients referred for investigation. We hoped to clarify the causes of inadequacy and to seek where the advantage lay with either method in patients with carcinoma of the oesophagus.

\section{Patients and methods}

The case notes of 404 patients with carcinoma of the oesophagus, referred over seven years to the Northern Ireland regional thoracic surgical unit, Royal Victoria Hospital, Belfast, were reviewed retrospectively. Two full time consultant surgeons and several registrars had performed the procedures. Those patients undergoing therapeutic oesophagoscopy have been excluded from study.

Three hundred and thirty six diagnostic oesophagoscopies were performed-165 procedures with the rigid oesophagoscope and 118 with the flexible oesophagoscope. Sixty three patients who had had flexible oesophagoscopy performed by consultants or registrars in other units but who had had no diagnosis made, tumour being suspected on other grounds, underwent rigid oesophagoscopy in our unit.

Rigid oesophagoscopy was performed under general anaesthesia and direct vision with a Negus rigid oesophagoscope. Flexible oesophagoscopy was performed under local analgesia and sedation with an Olympus flexible fibreoptic end viewing instrument. All diagnostic oesophagoscopies using either instrument were achieved without perforation. All patients had histological confirmation of carcinoma of the oesophagus, either 
Table 1 Results of biopsy and procedure in rigid and flexible oesophagoscopy

Successful diagnosis (No (\%))

\begin{tabular}{lrc}
\hline Rigid procedure & 165 & \\
Direct visualisation of lesion & 132 & $132(100)$ \\
Lesion suspected & 28 & $28(100)$ \\
No lesion suspected & 5 & $4(80)$ \\
Flexible procedure & 118 & $89(87)$ \\
Direct visualisation of lesion & 102 & $5(100)$ \\
Lesion suspected & 5 & $1(9)$ \\
No lesion suspected & 11 & \\
Rigid biopsy success rate (No (\%)) & $164(99.3)$ & $\mathrm{p}<0.02$ \\
Flexible biopsy success rate (No (\%)) & $95(80.5)$ & \\
\hline
\end{tabular}

as a result of the diagnostic procedure or subsequently at operation.

Where tumour was suspected visually, or where any observed mucosal feature that might be in keeping with the diagnosis was observed, multiple circumferential biopsy specimens were taken from above and within the lesion. In cases where the lesion could be passed, the distal oesophageal and stomach mucosa was examined. Specimens were immediately fixed in $10 \% \mathrm{w} / \mathrm{v}$ formalin and sent for routine histopathological examination.

Selection of patients for one procedure or the other was not randomised in this study and was determined by several factors. These included the patient's preference and compliance, the surgeon's preference, and availability of operating time. Previous investigations, such as a barium meal, in which stricture was noted, usually resulted in the selection of rigid oesophagoscopy so that, if feasible, therapeutic intervention remained an option. This applied particularly to older, high risk patients with advanced disease. The age range and the proportions of elderly high risk patients and of patients undergoing resection were similar in the groups having the two procedures.

Consultant surgeons in this unit are adept in the use of the fibreoptic instrument and carry out a weekly "day case" list to investigate a wide range of oesophageal diseases. Rigid instrumentation was performed by a consultant, or under the direct supervision of a consultant, except in the case of the senior registrar. In this study consultants performed

Table 2 Diagnostic details in 53 patients with no diagnosis from flexible oesophagoscopy who had a subsequent rigid procedure

\begin{tabular}{lrl}
\hline Direct visualisation of lesion & 35 & \\
Inadequate biopsy specimen & 12 & \\
Normal mucosa & 10 & \\
Dysplasia or metaplasia & 6 & \\
Non-specific changes & 7 & \\
No direct visualisation of lesion & 18 & \\
Inadequate biopsy specimen & 6 & \\
Normal mucosa & 9 & \\
Dysplasia or metaplasia & 2 & \\
Non-specific changes & 1 & \\
Subsequent rigid procedure & & Successful \\
& & diagnosis \\
Direct visualisation of lesion & 48 & 48 \\
No direct visualisation of lesion & 5 & 4 \\
\hline
\end{tabular}

43 procedures with the flexible instrument and directly supervised a further 30 . They performed 81 procedures with the rigid instrument and directly supervised 37 . The claim that surgeons are notorious for their "preference" for the rigid instrument and that examinations with the flexible instrument are practised by the less experienced junior staff, resulting in a case selection bias, has not been objectively tested but, given the above factors, such a selection bias is unlikely to have had a major influence on the results in this study.

Statistical analysis was by $\chi^{2}$ analysis, with Yates's correction where appropriate.

\section{Results}

In table 1 the findings in the groups having rigid and flexible oesophagoscopy are compared in the patients referred directly to our unit. Table 2 shows the findings in the 53 patients with failure of diagnosis in other units. The sites of tumour where biopsy reports were negative are shown in table 3.

\section{DIAGNOSTIC RIGID OESOPHAGOSCOPY}

A diagnostic procedure with the rigid oesophagoscope was performed in 165 patients without perforation. Where tumour was directly visualised ( $80 \%$ of cases) successful biopsy diagnosis of carcinoma was always obtained. There were 28 patients in whom a lesion was suspected but tumour could not be directly visualised because of a combination of stricture and proximal oedema. Successful biopsy was undertaken in all patients, including 20 in whom the neoplastic stricture was dilated. In four of five patients where a lesion was not suspected biopsy confirmation was obtained. The overall success rate of diagnosis with rigid oesophagoscopy was $99 \cdot 3 \%$. The patient who had a negative result from biopsy underwent surgical resection, which confirmed adenocarcinoma in situ. In all patients having the procedure with the rigid instrument adequate biopsy material was obtained for the pathologist to make a diagnosis.

\section{DIAGNOSTIC FLEXIBLE FIBREOPTIC \\ OESOPHAGOSCOPY}

A diagnostic procedure with the flexible oesophagoscope was performed in 118 patients without perforation. Where the tumour was directly visualised ( $86 \%$ of cases) biopsy confirmation of carcinoma was obtained in $87 \%$. In all five cases where a lesion was not visually identified but was suspected successful biopsy confirmation was

Table 3 Site of oesophageal tumour and result of biopsy

\begin{tabular}{lrl}
\hline Site of tumour & $n$ & $\begin{array}{l}\text { Negative biopsy result in } \\
\text { presence of tumour }\end{array}$ \\
\hline Upper third & 9 & 9 \\
Middle third & 113 & 6 \\
Lower third & 214 & 9 \\
\hline
\end{tabular}


obtained. In only one of 11 cases where no lesion could be identified was a successful biopsy report obtained. The overall success rate of diagnosis with flexible oesophagoscopy in this group was $80.5 \%$.

In those cases where tumour was directly visualised there were 13 failures; eight of these were due to inadequacy of the material $(61 \%)$, whereas in five the report was nonspecific. Among those where a lesion was not identified visually material was inadequate for diagnosis in two (18\%) whereas normal mucosa was reported in the remainder, indicating failure to obtain biopsy material from the tumour itself.

When the success rates of diagnosis of tumour were compared in the rigid group and the flexible group a significantly better result was achieved in the former $(p<0.02)$.

\section{RIGID OESOPHAGOSCOPY AFTER FLEXIBLE OESOPHAGOSCOPY}

The rigid oesophagoscope was used in 53 patients who had previously undergone flexible oesophagoscopy with no positive diagnosis of tumour made. Results are summarised in table 2. When this same group underwent rigid oesophagoscopy there were no perforations and tumour was directly visualised in 48 cases $(90 \%)$.

Direct visualisation of the lesion was achieved significantly more often with the rigid oesophagoscope in this group of patients than by other workers with the flexible instrument $(\mathrm{p}<0.05)$.

\section{Discussion}

In this study of 336 patients we used the objective measures of rate of perforation and diagnostic efficacy in patients with carcinoma of the oesophagus to compare procedures using rigid and flexible fibreoptic oesophagoscopes. Perforation of the oesophagus has previously been used as an objective measure of the success of oesophagoscopy and led to claims that the flexible instrument was superior in this regard. ${ }^{289}$ We have shown that when used for diagnosis alone rigid and flexible instruments can achieve their results with no perforation. Our results differ from those of others, who claim that flexible oesophagoscopy carries less risk. ${ }^{8}$ The premises used to make such comparisons are unsound because many reports do not make a clear distinction between diagnostic and therapeutic use. Even in those that do, study groups have included patients without malignant disease and those where a normal oesophagus was examined en route to the stomach. ${ }^{8}$ Only one study has directly compared the instruments in patients with early disease who had endoscopy with a view to surgical resection and who were at less risk than those in the present study. ${ }^{2}$ These comparisons have failed to evaluate the increased risk recognised with oesophagoscopy of malignant strictures, which is not comparable to the risk of endoscopy in a normal oesophagus.
The higher success rate of diagnosis of carcinoma with the rigid instrument $(99 \%)$ than with the flexible instrument $(80 \cdot 5 \%)$ is significant in our group of patients $(p<0.02)$. This could be explained by intrinsic differences between the two groups of patients, but such an explanation is unlikely. Direct visualisation of the lesion was obtained more often with the rigid than with the flexible instrument, and where this is achieved with either instrument biopsy is usually successful. The larger and deeper biopsy specimens obtainable with the rigid instrument are likely to increase the number of submucosal tumour specimens biopsied. The increased number of normal mucosal specimens seen with flexible biopsy suggests that small bite sampling of a wide area is less successful, especially where no obvious lesion has been identified.

These results are substantiated by the findings in the 53 patients referred to our unit as a result of failure to obtain a diagnosis in other units. The consultants and registrars performing the procedures in the other units and our own have not been objectively assessed in this study but were similar in terms of seniority and experience. Whether the difference is due to greater skill of the surgeon than of the physician in using the flexible instrument or to the limitations of the instrument itself would be an interesting subject for a randomised clinical trial. The latter is suggested by our results as there had been significantly less direct visualisation of the lesion with the flexible oesophagoscope in this group when the procedure has been performed by other units, which had at least as much experience with the flexible instrument as we had. The delay of less than a month between one procedure and the other is unlikely to account for this. Inadequate biopsy material was obtained in $34 \%$ and normal mucosa in $36 \%$. Together these account for $70 \%$ of the failures. The same group of patients undergoing rigid instrumentation in our unit had a greater number of direct visualisations of the lesion. Although this difference does not reach statistical significance, we have shown earlier that direct visualisation is associated with an increased positive biopsy rate in this group of patients.

In conclusion, we have presented data showing that diagnostic oesophagoscopy can be achieved without causing deaths with either rigid or flexible instruments in this high risk group of patients and that use of the rigid instrument is associated with a significantly greater chance of positive diagnosis than the flexible instrument. We suggest that this is likely to be related to increased direct visualisation of lesions. Claims that the fibreoptic instrument is superior may be more apparent than real as no prospective randomised clinical trial comparing the instruments in a diagnostic setting in such high risk groups has been performed.

We wish to thank Miss May Weller, who typed the manucript. AJR is indebted to the Northern Ireland Chest, Heart, and Stroke Society and the Royal Victorit 
1 Steiger Z, Franklin R, Wilson RF. Eradication and palliation of squamous cell carcinoma of the oesophagus with chemotherapy, radiotherapy and surgical therapy. $\mathcal{F}$ Thorac Cardiovasc Surg 1981;82:713-9.

2 Cheung HC, Siu KF, Wong J. A comparison of flexible and rigid endoscopy in evaluating esophageal cancer patients for surgery. World ₹ Surg 1988;12:117-22.

3 Hartley L, Strong R, Fielding G, Evans E. Morbidity and mortality of operative intubation for malignant oesophageal obstruction. Aust $N Z$ f Surg 1985;55: 555-7.

4 Moghissi K, Pender D. Instrumental perforations of the oesophagus and their management. Thorax 1988;43: 642-6.

5 Nashef SAM, Pagliero KM. Instrumental perforation of the esophagus in benign disease. Ann Thorac Surg 1987;44:360-2.

6 Moghissi K. Instrumental perforations of the oesophagus.
Br $\mathcal{H}$ Hosp Med 1988;39:231-6.

7 Lishman AH, Dellipiani AW, Devlin HB. The insertion of oesophagogastric tube in malignant oesophageal strictures: endoscopy or surgery? $B r f$ Surg 1980;67: 257-9.

8 Dawson J, Cockel R. Oesophageal perforation at fibreoptic gastroscopy. BMF 1981;283:583.

9 Hine KR, Atkinson M. The diagnosis and management of perforations of esophagus and pharynx sustained during intubation of neoplastic esophageal strictures. Dig Dis Sci 1986;31:571-3.

10 Wesdorf ICE, Bartelsman JFWM, Den Hartog Jager FCA, et al. Results of conservative treatment of benign FCA, et al. Results of conservative treatment of benign
oesophageal strictures: a follow-up study in 100 patients. Gastroenterology 1982;82:487-93.

11 Bennet JR, Hendrix TR. Treatment of achalasia with pneumatic dilatation. Modern Treatment 1970;7: 1217-24. 